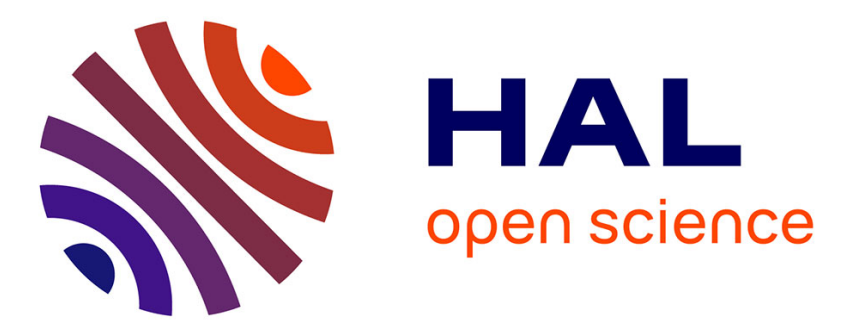

\title{
A nonlinear resonant mass sensor with enhanced sensitivity and resolution incorporating compressed bistable beam
}

Jian Zhao, Chen Sun, Najib Kacem, Hongxi Wang, Renjing Gao, Pengbo Liu, Yu Huang

\section{To cite this version:}

Jian Zhao, Chen Sun, Najib Kacem, Hongxi Wang, Renjing Gao, et al.. A nonlinear resonant mass sensor with enhanced sensitivity and resolution incorporating compressed bistable beam. Journal of Applied Physics, 2018, 124 (16), pp.164503. hal-02130043

\section{HAL Id: hal-02130043 \\ https://hal.science/hal-02130043}

Submitted on 15 May 2019

HAL is a multi-disciplinary open access archive for the deposit and dissemination of scientific research documents, whether they are published or not. The documents may come from teaching and research institutions in France or abroad, or from public or private research centers.
L'archive ouverte pluridisciplinaire HAL, est destinée au dépôt et à la diffusion de documents scientifiques de niveau recherche, publiés ou non, émanant des établissements d'enseignement et de recherche français ou étrangers, des laboratoires publics ou privés. 


\title{
A nonlinear resonant mass sensor with enhanced sensitivity and resolution incorporating compressed bistable beam
}

\author{
Jian Zhao ${ }^{1, \text { a) }}$, Chen Sun ${ }^{1}$, Najib Kacem ${ }^{2}$, Hongxi Wang ${ }^{3}$, Renjing Gao ${ }^{1}$, Pengbo Liu ${ }^{1}$, Yu Huang ${ }^{4}$ \\ ${ }^{1}$ State Key Laboratory of Structural Analysis for Industrial Equipment, Dalian University of \\ Technology, 116024, Dalian, China \\ ${ }^{2}$ Univ. Bourgogne Franche-Comté, FEMTO-ST Institute, UMR 6174, \\ CNRS/UFC/ENSMM/UTBM, Department of Applied Mechanics, F-25000, Besançon, France \\ ${ }^{3}$ School of Mechatronics, Xi'an Technological University, 710021, Xi'an, China \\ ${ }^{4}$ College of Ocean and Civil Engineering, Dalian Ocean University, 116023, Dalian, China
}

\begin{abstract}
:
A new piezoelectric actuated nonlinear mass sensor is proposed by using the snap-through phenomenon of compressed bistable beam to enhance the sensitivity and resolution, which can be used to weigh or detect threshold mass by tracking the bifurcation frequency shift. According to the nonlinear finite element modeling technique, the nonlinear dynamic response of the combined nonlinear structure is numerically calculated, which shows that the bifurcation point can be accurately identified by the sharp and great amplitude change regardless of the damping effect, thus providing an effective way for tracking the bifurcation frequency. Hence, the nonlinear sensitivity depicted by the bifurcation frequency shift per unit mass can reach 3.3 times the sensitivity of linear mass sensors having the same size. Also, as a mass switch, the response amplitude jumps sharply when the added mass is greater than or equal to the threshold value, which is depending on the excitation frequency. Meanwhile, the influences of the beam compression and excitation voltage on the sensitivity and minimum detectable mass were obtained for sensor optimization. For concept validation, a macro-sized nonlinear mass sensor was fabricated with the geometric size of $58.0 \mathrm{~mm}$ long and $4.0 \mathrm{~mm}$ wide, and the experimental results show that the sensitivity is around $575.0 \mathrm{~Hz} / \mathrm{g}$ compared with the simulated sensitivity of $542.0 \mathrm{~Hz} / \mathrm{g}$. For mass switch, the minimum threshold mass is $0.2 \mathrm{mg}$. The fair agreement between the simulation and experiments adequately validated the proposed nonlinear bistable mass sensor.
\end{abstract}

\section{Introduction}

Resonant cantilever mass sensors can quantitatively detect the unknown analyte by measuring the induced frequency shift or amplitude change at resonance. These sensors have been successfully applied in various application fields ${ }^{1}$, such as biosensing ${ }^{2}$, gas density $^{3}$, and chemical or fluidic detection ${ }^{4}$. Utilizing the micro-nano manufacturing technology, mass sensors have been attributed with the capability of detecting atom or even small nanoparticles in vacuum ${ }^{5}$. However, in fluid or air environments, such miro/nano-machined sensors are greatly limited by the energy loss in the dynamic regime ${ }^{6-8}$, especially the damping effect, which will directly reduce the resolution for tracking the resonant frequency distinguished by the peak response amplitude ${ }^{9-11}$. Therefore, exploring effective methods to avoid or reduce the

a)jzhao@dlut.edu.cn damping effects is of great importance for resonant mass sensors.

In recent years, a series of new mass sensing methods have been proposed by introducing the bifurcation phenomenon in nonlinear structures ${ }^{12-}$ 16. Piezoelectrically-actuated cantilevers are well suited for resonant mass sensing with capabilities of self-sensing and actuating. Kumar et al. ${ }^{12,13}$ proposed a piezoelectric resonant chemical and biological sensor by using the dynamic transitions across saddle-node bifurcations, which is really an amplitude-based mass sensing method rather than that of tracking the frequency shift. Bouchaala et $a l .{ }^{17,18}$ presented a nonlinear gas sensor by using the nonlinear response of micromachined clamped-clamped beams coated with metalorganic framework (MOF), which can perform two functionalities of mass sensor and switch triggered by predefined threshold. Besides piezoelectric driving, electrostatic actuation has also been introduced in nonlinear sensors. Sansa et $a l .^{7,19}$ have proposed a nonlinear mass detection technique in real time by using the bifurcation of 
the electrostatic actuated beam, and presented a frequency modulation measurement method to avoid the re-setting procedure after each sensing event. Harne and Wang $^{20}$ proposed a robust sensing methodology utilizing bistable circuitry to tailor dynamics, which greatly enhances the versatility of bifurcation-based sensing. Zhang et $a{ }^{21}$ designed a nonlinear mass sensor based on parametric resonance amplification, which is experimentally validated by a non-interdigitated comb-finger driven micro-oscillator. Kacem et al. 22 proposed a dynamic stabilization method for high order nonlinearity cancellation by simultaneous using primary and super harmonic resonances, which provides a new way for high frequency resonators to be used as mass sensing or time referencing devices. Prakash et al. ${ }^{23}$ used an electronic feedback circuit to make the micro cantilever working in both regimes of parametric resonance and noise squeezing which can improve the quality factor by two orders of magnitude under ambient conditions, thus opening the field to high sensitivity mass sensing in liquid environments. Furthermore, Li et al. ${ }^{24}$ proposed a method for detecting explosive gas (DNT) by using the noise squeezing effects that occurs before a bifurcation event. With these nonlinear methods, both the response amplitude and the bifurcation frequency shift can be used to measure external perturbations like additional mass or force, especially the sharp amplitude change is helpful for reducing the damping effects on the resolution. Known from the snap-through procedure of bistable structures ${ }^{\mathbf{2 5}, \mathbf{2 6}}$, a sharp amplitude change can be achieved when the stable state switching is triggered. So, they are regarded as ideal candidates for nonlinear spring elements in sensors. The main difficulty lies in the lack of method to use the mass change to trigger the bistable structure in dynamic mode.

In this paper, a new piezoelectric nonlinear mass sensor is proposed by using the bistability induced bifurcation phenomenon, which processes both functions of mass weighing and threshold mass detection. The sensor mainly consists of linear piezoelectric-actuated cantilever and nonlinear compressed bistable beam. With the nonlinear finite element method, the dynamics of the combined structure is numerically calculated. One can accurately identify the bifurcation frequency by measuring the sharp change of the response amplitude, which can significantly reduce the damping effect on the frequency resolution. By tracking the bifurcation frequency shift induced by the added mass, the sensitivity can be determined. Compared to linear mass sensors, a significant enhancement of sensitivity is highlighted. Also, the influences of the excitation frequency and voltage on the bifurcation frequency, sensitivity and the minimum detectable threshold mass are analyzed. Finally, a macrosized nonlinear sensor is fabricated and the proposed concept of mass sensing is experimentally validated.

\section{Nonlinear mass sensor with compressed bistable beam}

\subsection{Sensor structure}

A new nonlinear resonant mass sensor is proposed for both mass weighing and threshold mass detection, as shown in Fig.1, which mainly consists of one piezoelectric layer, one elastic cantilever, one compressed bistable beam, and one piezoelectric patch placed near the cantilever fixed end. The soft film marked in black is used to absorb the external analyte. Fully compliant bistable mechanisms ${ }^{\mathbf{2 5}, \mathbf{2 6}}$ have been widely used as sensor spring elements due to their snapping through characteristic. In this paper, the proposed sensor is a complex composite structure involving several separate parts, especially, the bistable beam fixed on the surface of the cantilever. Therefore, the structure simplicity and easy fabrication are two decisive factors for selecting right bistable elements for practical applications. In this design, the selected uniform beam can be attributed with bistability by just compression without any extra manufacturing processes for configuration modification. And the piezoelectric layer is used to drive the mass sensor to in its nonlinear dynamic range.

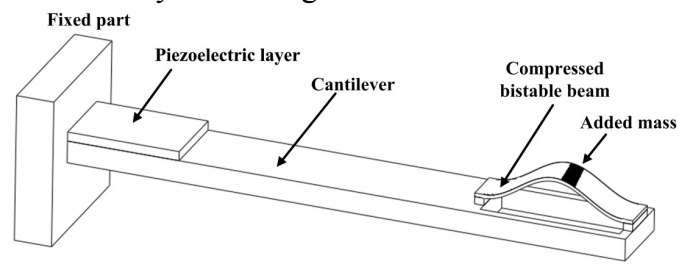

Fig.1 The bistability based mass sensor

According to the vibration theory, the bifurcation frequency and dynamic response will be changed when an external mass is added to the beam surface. Especially, a sharp change in response amplitude will occur when the bifurcation frequency is tuned close to the excitation frequency by the added mass. Then, the corresponding bifurcation frequency shift can be 
measured and recorded by the laser sensor and data acquisition circuits, enabling the determination of the added mass. Actually, this bifurcation frequency shift is obtained by sweeping the frequency of the harmonic excitation. Also, the sensor performance can be adjusted by simultaneously changing the excitation voltage, structure parameters, and compressions. With the obtained frequency variation, the mass detection sensitivity can be calculated as follows:

$$
S=\frac{\Delta f}{\Delta m}=\frac{f_{b}-f_{a}}{\Delta m}=\frac{\Delta \omega}{2 \pi \Delta m}
$$

Where $f_{b}$ and $f_{a}$ are the bifurcation frequencies of the sensor before and after absorbing mass, and $\Delta f$ is the corresponding frequency shift induced by the mass change $\Delta m$.

In order to measure accurately the added mass, the induced bifurcation frequency shift should be precisely determined. In practice, the resonator will be embedded in a phase locked loop (PLL) or a self-excited loop in order to monitor time evolution of the bifurcation point frequency, enabling an accurate measure of the frequency shift.

The minimum measurable frequency shift $\Delta \omega$ can be obtained by integrating the weighted effective spectral density of the frequency fluctuations $S_{\omega}(\omega)$ by the normalized transfer function of the measurement loop $H(\omega)^{27,28}$

$$
\Delta \omega=\left[\int_{0}^{\infty} S_{\omega}(\omega) H(\omega) d \omega\right]^{1 / 2}
$$

Following Robins ${ }^{\mathbf{2 9}}$, the frequency fluctuations is given by

$$
S_{\omega}(\omega)=\left(\frac{\omega_{n}}{2 Q}\right)^{2} \frac{S_{x}(\omega)}{E_{c}}
$$

where $S_{x}(\omega)$ is the displacement spectral density, $E_{c}$ is the output displacement carrier power defined as the RMS drive amplitude of the sensor, $Q$ the quality factor, and $\omega_{n}$ the natural frequency. The quality factor is a measure of the sharpness of the frequency selectivity of the resonator which is given by the ratio between the resonance frequency and the half-power bandwidth.

From equations (2) and (3), driving the sensor at large amplitudes can significantly reduce the frequency spectral density $S_{\omega}(\omega)$. Consequently, the minimum measurable frequency shift $\Delta \omega$ will be decreased, which will induce a smaller detectable mass $\Delta m$. In this paper and unlike linear cantilevers, the snap-through phenomenon can significantly amplify the response amplitude of the sensor, and thus resulting in an enhanced mass resolution.

Tab.1 Parameters of the stepped cantilever sensor

\begin{tabular}{llcc}
\hline Type & $\begin{array}{c}\text { Piezoelectric } \\
\text { layer }\end{array}$ & $\begin{array}{c}\text { Elastic } \\
\text { cantilever }\end{array}$ & $\begin{array}{c}\text { Bistable } \\
\text { beam }\end{array}$ \\
\hline Length $(\mathrm{mm})$ & 12.0 & 58.0 & 22.0 \\
Thickness $(\mathrm{mm})$ & 0.2 & 0.2 & 0.02 \\
Width $(\mathrm{mm})$ & 4.0 & 4.0 & 1.0 \\
Density $\left(\mathrm{Kg} / \mathrm{m}^{3}\right)$ & $7.5 \times 10^{3}$ & $8.3 \times 10^{3}$ & $8.3 \times 10^{3}$ \\
Poisson ratio & 0.32 & 0.35 & 0.35 \\
$\quad$ Elastic & 76.5 & 128.0 & 128.0 \\
modulus $(\mathrm{GPa})$ & & & \\
\hline
\end{tabular}

With the parameters listed in Table 1, the dynamic response of the proposed nonlinear bistable mass sensor is numerically obtained, as shown in Fig.2, and the detailed simulation process will be depicted in the next section. It can be seen that the response amplitude of the nonlinear bistable sensor changes sharply at the bifurcation point due to the snap-through phenomenon compared to the linear cantilever sensor having the same size, thus resulting in a higher frequency resolution. In fact, the response amplitude of linear mass sensor suffers from the damping effects with low quality factor in air, which implies a poor mass resolution. From equation (3), it is known that improving the response amplitude will significantly increase the output carrier power, and then, decrease the minimum detectable frequency shift. With the nonlinear bistable structure in this paper, the response amplitude can be significantly amplified compared to linear cantilever sensors under the same excitation as shown in Fig.2. Therefore, the bifurcation frequency can be accurately determined on the upper branch with a sharp change in amplitude where the transduction gain is high, which results in a high mass resolution.

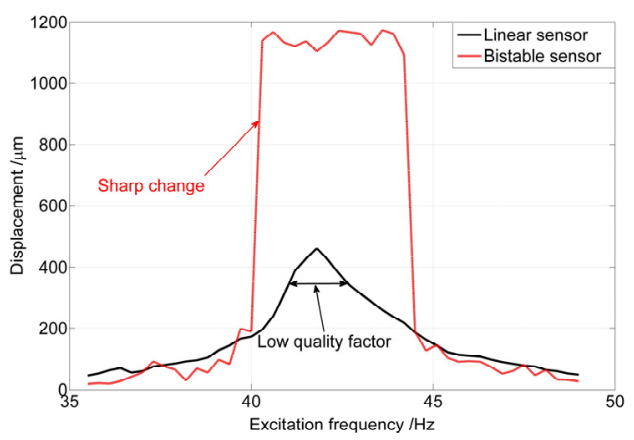

Fig.2 Frequency response comparison for linear and nonlinear sensor 


\subsection{Dynamic simulations}

Due to the strongly nonlinear characteristics of the snap-through procedure, it is difficult to analytically calculate the frequency response of the combined nonlinear structure. Here, the nonlinear Finite Element (FE) analysis has been used to predict the bistable mechanics as well as its nonlinear dynamics. So, the FE model of the nonlinear mass sensor is developed using ABAQUS (Multiphysics, Abaqus/Explicit), and the beam is modeled using B21 Timoshenko beam element. The piezoelectric structure is made up of PZT-5H (Lead Zirconate Titanate) and is modeled using the 20 node quadratic hexagon piezoelectric C3D20E solid elements with an additional degree of freedom for electric potential. Transversely isotropic material properties are assigned to the piezoelectric layer including dielectric and piezoelectric properties, and the relative dielectric constant is 3800 and the permittivity of free space is $8.85 \times 10^{-12} \mathrm{~F} / \mathrm{m}$. The constitutive equations in the e-form of the piezoelectric material expressed in the stress format are

$$
\left\{\begin{array}{l}
\sigma_{i j}=D_{i j k l}^{E} \varepsilon_{k l}-e_{m k l}^{\varphi} E_{m} \\
q_{j}=e_{i j k}^{\varphi} \varepsilon_{j k}+D_{i j}^{\varphi} E_{j}
\end{array}\right.
$$

where $\sigma_{i j}$ is the stress matrix, $q_{j}$ is the vector of electric displacement, $\varepsilon_{k l}$ is the strain vector, $D^{E} i j k l$ is the matrix of elastic coefficients, $e^{\varphi}{ }_{m k l}$ is the stress piezoelectric matrix, $D^{\varphi} i j$ is the electric matrix with the coefficients of electric permittivity on its diagonal.

The dynamic analysis of the combined nonlinear structure includes two main steps that one is the pre-stressing stage to compress the straight beam to a curved configuration, and the other is the simulation of the frequency response of the combined nonlinear structure. In the first stage, the stress field induced by the axial compression can directly result in the required bistability in the transverse direction. In the second stage, the compressed beam is fixed on the free end of the linear cantilever, as shown in Fig.1. Then, the nonlinear dynamic response of the combined structure is obtained under different excitations from the piezoelectric layer through harmonic response analysis. When the bifurcation phenomenon occurs, the response amplitude increases sharply compared with that of the linear cantilever. Thereafter, the bifurcation frequencies before and after adding external mass can be determined by sweeping the excitation frequency in sinusoidal form.
Through tracking its response amplitude for determining the bifurcation point, the related frequency shifts induced by different masses can be numerically obtained as shown in Fig.3. In this paper, the harmonic excitation with the voltage of $27.0 \mathrm{~V}$ is used to drive the piezoelectric layer. In Fig.3(a), while the added mass is $500 \mu \mathrm{g}$, the bifurcation frequency shift of the combined structure is $0.271 \mathrm{~Hz}$ changing from $40.1 \mathrm{~Hz}$ to $39.83 \mathrm{~Hz}$ compared with frequency shift of 0.082 $\mathrm{Hz}$ from the linear resonant cantilever mass sensor with the same geometric size. At the bifurcation point on the amplitude-frequency curve, the response amplitude changes sharply from nearly $200.0 \mu \mathrm{m}$ to the maximum of $1180.0 \mu \mathrm{m}$, which makes it easier to distinguish the frequency shift. For threshold mass detection, the mass sensor is used as a switch, which is actuated at a fixed excitation frequency near the bifurcation frequency. When the mass is loaded on the sensor surface, the dynamic response change in time domain is numerically calculated as shown in Fig.3(b). It can be seen that the amplitude is very small while no mass is added to the sensor. When the threshold mass is loaded, the structure's effective mass increases and thus, its bifurcation frequency decreases to the value close to the excitation frequency. When the bifurcation occurs, the extreme large response amplitude is triggered like the state changing of a switch. So, the mass sensor can be used as a threshold mass switch for detecting threshold masses.

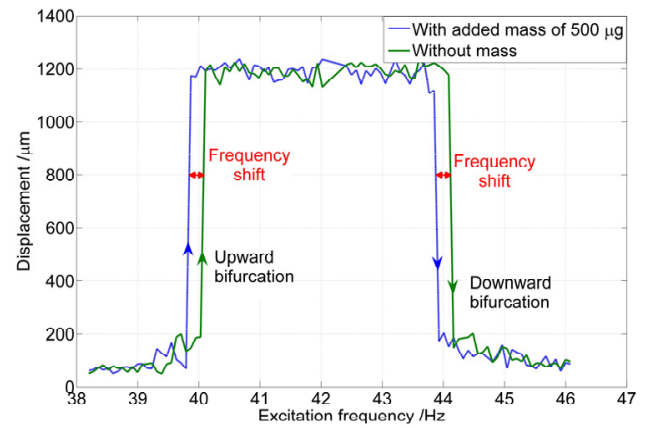

(a)

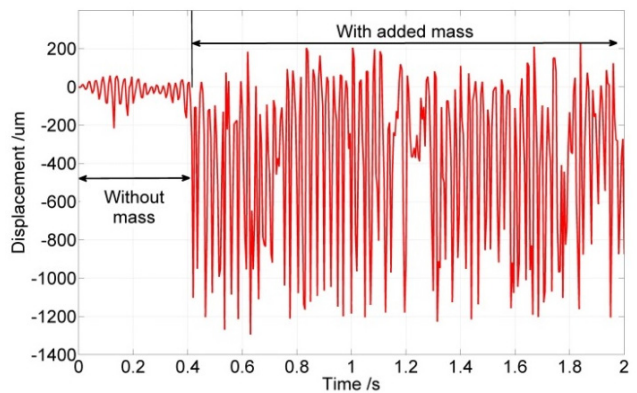

(b) 
Fig. 3 Dynamic response of the nonlinear mass sensor, (a) Frequency response for mass measuring, (b) Mass induced dynamic response in time domain

Actually, as a mass switch, the minimum detectable mass is highly dependent on the difference between the excitation frequency and the bifurcation frequency of the original nonlinear structure without loading mass. Figure 4 shows the relationship between the excitation frequency and the detectable threshold mass. It can be seen that the nonlinear sensor operates at a large amplitude vibration when the added mass is greater than or equal to the threshold value. When the excitation frequency is $39.89 \mathrm{~Hz}$, the minimum threshold mass of the switch can reach $0.3 \mathrm{mg}$, which also means that all added masses greater than $0.3 \mathrm{mg}$ will definitely trigger the mass switch. To further minimize the detection threshold value, the counterweight mass can be placed on the beam surface, and the difference between the original threshold value and the external counterweight mass can be the new minimum detectable threshold mass of the switch. Overall, it is applicable to use the proposed nonlinear sensor to weigh or detect the unknown mass and threshold analyte by using different measuring strategies.

Furthermore, the influences of the beam compression and excitation voltage on both the sensitivity and mass threshold have been obtained, as shown in Fig.5 and Fig.6. For mass weighing as shown in Fig.5, it can be seen that the sensitivity can be enhanced by increasing the excitation voltage and/or increasing the compression, which results in strengthening the bistablity domain. As a mass switch, the threshold mass is also influenced by the compression and excitation voltage, as shown in Fig.6. Notably, Figures 5 and 6 show that tuning the excitation voltage around $30.0 \mathrm{~V}$ and the compression rate around $35.0 \mu \mathrm{m}$ leads to an optimal configuration in terms of performances with a high sensitivity and a low mass threshold.

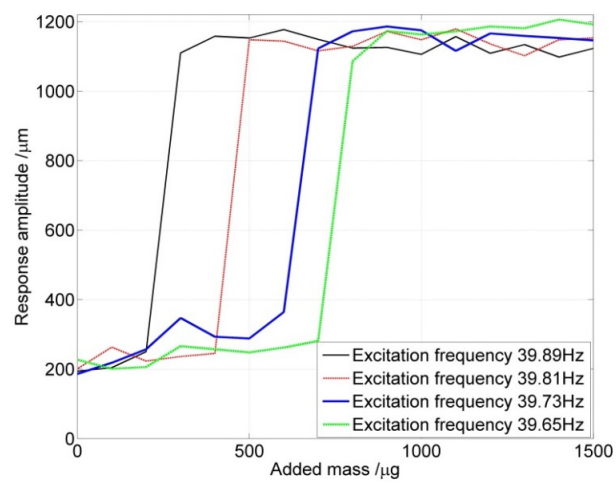

Fig. 4 The bifurcation frequency shift versus the added mass

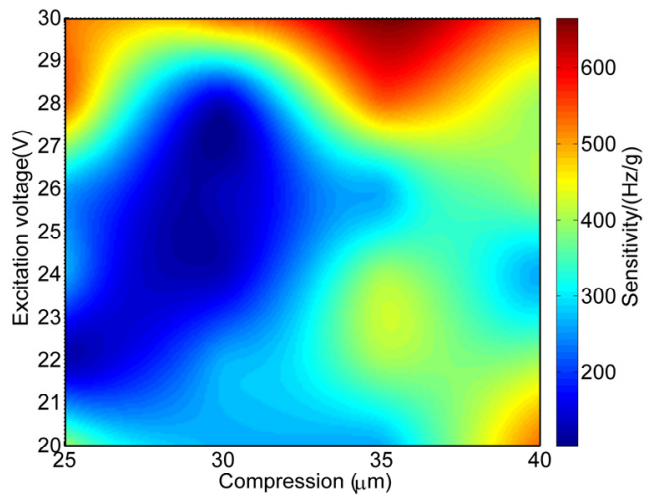

Fig.5 Influences of compression and excitation on sensitivity

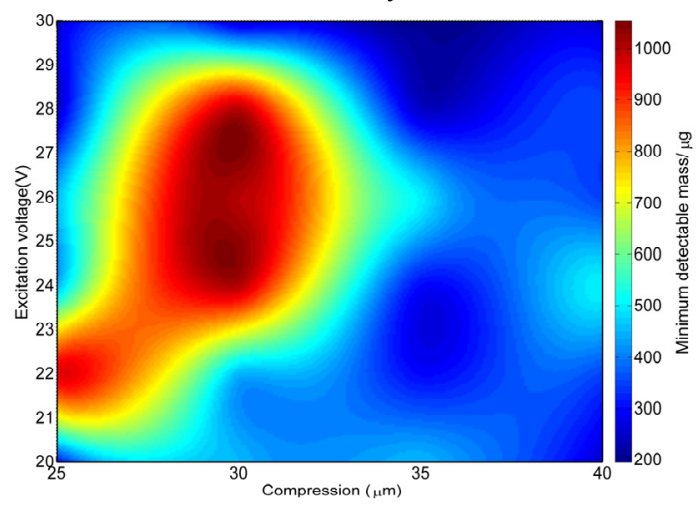

Fig.6 Influences of compression and excitation on mass threshold

\section{Experiments}

To validate the concept of the nonlinear mass sensing, a macro-sized nonlinear mass sensor with bistable structure is fabricated as shown in Fig.7, and the test setup is also established for measuring the sensor response in Figure 8. The main fabrication procedure includes three steps. Firstly, a straight beam is compressed to its buckled state to achieve bistability. Secondly, both ends of the buckled beam are stuck to the surface of the cantilever near the free end. Thirdly, the piezoelectric layer is stuck to the upper surface of the cantilever near the fixed end, as shown in Fig.7. The testing setup mainly consists of one oscilloscope, signal generator, voltage amplifier, laser displacement sensor, and the computer based data acquisition system. For actuating the piezoelectric layer, the harmonic signal is amplified up to the amplitude of $27.0 \mathrm{~V}$ through the voltage amplifier. Meanwhile, the response amplitude of the piezoelectric nonlinear sensor is measured by the laser displacement sensor, as shown in Fig.9.

It can be seen that the simulated upward bifurcation frequency of $40.1 \mathrm{~Hz}$ is a bit lower than the experimental one located at $40.4 \mathrm{~Hz}$, and also the downward bifurcation frequency of 44.2 $\mathrm{Hz}$ is a bit higher than the experimental one located at $43.6 \mathrm{~Hz}$. The slight differences between 
the experiments and simulations are possibly due to the complex interactions among the compression deviation, damping effects, the existence of bonding layer between the piezoelectric layer and elastic cantilever, and temperature affecting the piezoelectric capacitance. For the case of the upward bifurcation, the structure stiffness plays a main role in increasing the bifurcation frequency. For the downward bifurcation, the response is already at a high amplitude and meanwhile, the damping effects increase. So the downward bifurcation decreases in experiments. Finally, while considering the manufacturing errors, the experimental and numerical results are in good agreement, which validates the proposed concept of nonlinear mass sensing.

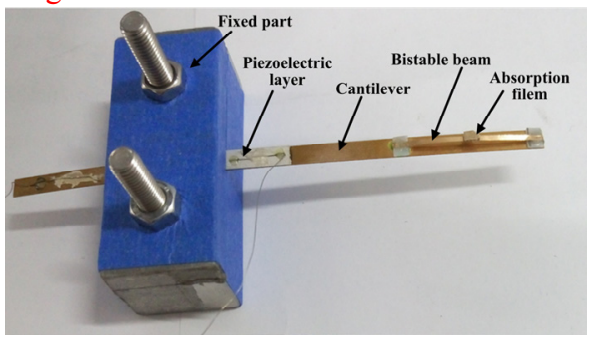

Fig.7 Piezoelectric nonlinear mass sensor

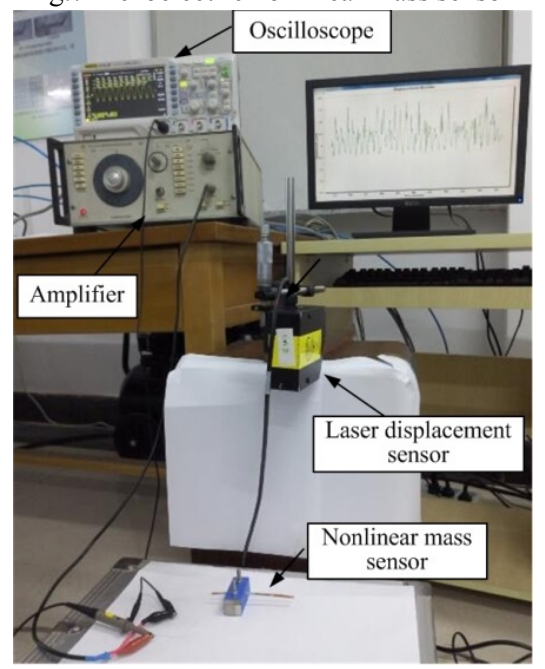

Fig.8 Experiments setup

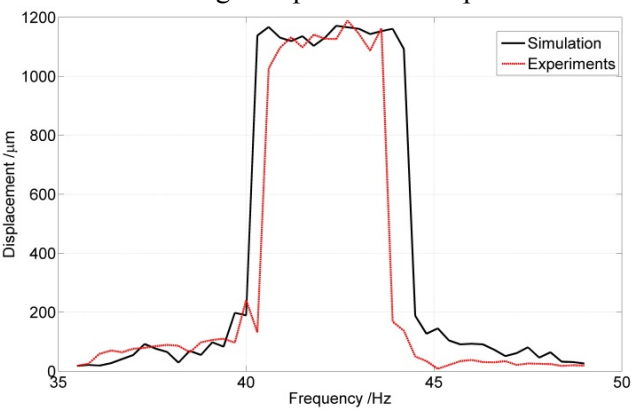

Fig.9 Comparison of the simulated frequency response with experiments

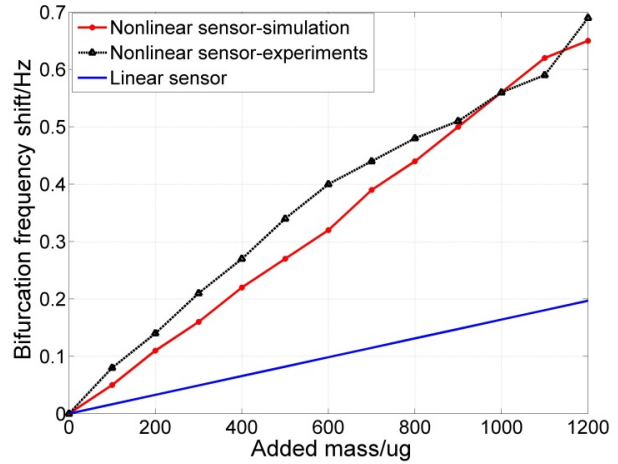

Fig.10 Sensitivity comparison between experiment and simulation

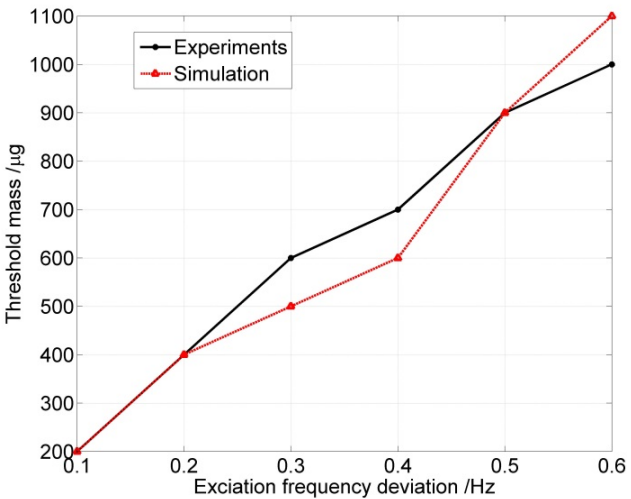

Fig.11 Threshold mass affected by excitation frequency

In experiments, the upward bifurcation frequency is selected to weigh added masses, and the experimental sensitivity is obtained in Fig. 10. The experimental sensitivity can reach about $575.0 \mathrm{~Hz} / \mathrm{g}$ compared with the simulated sensitivity of $542.0 \mathrm{~Hz} / \mathrm{g}$, which is nearly 3.3 times the sensitivity of a linear mass sensor with the same size. Also, the sensitivity simulations are in agreement with the experimental results with a maximum deviation of $6.0 \%$. The relatively higher experimental sensitivity compared to that of the simulation is mainly due to the higher upward bifurcation sensitivity.

For threshold mass detection, the influence of the excitation frequency on the threshold value has been experimental analyzed, as shown in Fig.11. It can be seen that the minimum detectable mass is highly dependent on the deviation of the excitation frequency from the non-mass loaded bifurcation frequency, and the threshold mass increases with the frequency deviation. For instance, when the deviation frequency is $0.1 \mathrm{~Hz}$, the minimum detectable threshold mass is $0.2 \mathrm{mg}$.

This nonlinear sensing device is a first step for utilizing compressed bistable beams in mass detection, which provides a way of using snapthrough procedure to enhance the frequency resolution at the bifurcation point compared to 
linear mass sensors. Actually, the robustness to noise and damping of the proposed nonlinear bistable sensor is important for its further practical applications ${ }^{27}$, and several excellent works have been published addressing the noise sensitivity and noise induced bifurcations ${ }^{19},{ }^{29-35}$, which will be needed but beyond the goal of this paper.

\section{Conclusion}

A new piezoelectric nonlinear mass sensor is proposed with both functions of mass weighing by frequency shift and threshold mass sensing through bistable state switching, which mainly consists of one compressed bistable beam and one piezoelectric-actuated cantilever.

(1) By using the bistability induced bifurcation phenomenon, the influence of damping dissipation on the frequency resolution can be significantly reduced, and the nonlinear mass detection sensitivity has been significantly improved up to 3.3 times more sensitive than linear mass sensors having the same size. Actually, the inclination of the frequency-amplitude curve at the bifurcation point is about 6.6 times greater than that of the linear sensor, which guarantees a high frequency resolution for mass sensing.

(2) According to the nonlinear finite element modeling technique, the nonlinear dynamic response of the proposed sensor is numerically calculated, and also, the influences of the excitation frequency and excitation voltage on the bifurcation frequency, sensitivity and minimum detectable threshold mass were obtained. When the added mass is greater than or equal to the threshold value, the response amplitude at the bifurcation frequency jumps sharply.

(3) The two functions of mass weighing and threshold mass detection can be switched by using different measuring strategies including the frequency sweeping method and excitation at fixed frequency near the bifurcation point.

For concept validation, a macro-sized nonlinear sensor was fabricated with the size of $58.0 \mathrm{~mm}$ long and $4.0 \mathrm{~mm}$ wide. The experimental results show that the sensitivity for mass weighing is $575.0 \mathrm{~Hz} / \mathrm{g}$ compared with the simulated sensitivity of $542.0 \mathrm{~Hz} / \mathrm{g}$, and the minimum threshold mass is $0.2 \mathrm{mg}$ which is also depending on the difference between the excitation frequency and the bifurcation frequency. The agreement between the simulations and the experiments adequately validated the proposed nonlinear bistable mass sensor.

\section{Supplementary Material}

See supplementary material for structure of the bistability based mass sensor, frequency response comparison for linear and nonlinear sensor, dynamic response of the nonlinear mass sensor including frequency response for mass measuring and mass induced dynamic response in time domain, the bifurcation frequency shift versus the added mass, influences of compression and excitation on sensitivity, influences of compression and excitation on mass threshold, piezoelectric nonlinear mass sensor, experiments setup, comparison of the simulated frequency response with experiments, sensitivity comparison between experiment and simulation, and threshold mass affected by excitation frequency.

\section{Acknowledgments}

This work was supported in part by the National Natural Science Foundation of China (Grant No. 51575088, 11502043, 61874019), and the Fundamental Research Funds for the Central Universities. This project is also performed in cooperation with EUR EIPHI program (contract ANR 17-EURE-0002).

\section{References}

A. Boisen, S. Dohn, S. S. Keller, S. Schmid and M. Tenje. Cantilever-like micromechanical sensors. Rep. Prog. Phys. 74, 036101 (2011).

B. Johnson, R. Mutharasan. Biosensing using dynamic-mode cantilever sensors: A Review. Biosens. Bioelectron. 32, 1-18 (2012).

G. Popescu, K. Park, M. Mir, R. Bashir. New technologies for measuring single cell mass. Lab. Chip. 14, 646-652 (2014).

L. Zhao, Y. Hu, T. Wang, J. Ding, X. Liu, Y. Zhao and Z. Jiang. A MEMS Resonant Sensor to Measure Fluid Density and Viscosity under Flexural and Torsional Vibrating Modes. Sensors. 16(6), 830 (2016).

K. Jensen, K. Kim and A. Zettl. An atomic-resolution nanomechanical mass sensor. Nat. Nanotechnol. 3, 533-537 (2008).

A. N. Cleland and M. L. Roukes. Noise processes in nanomechnical resonators. J. App. Phys. 92(5), 2758-2769 (2002).

M. Sansa, V-N. Nguyen, S. Baguet, C-H. Lamarque, R. Dufour, and S. Hentz. Real time sensing in the nonlinear regime of NEMS resonators. MEMS 2016, Shanghai, China, 24-28 January, 2016.

H W C Postma, I Kozinsky, A Husain, and M L Roukes. Dynamic range of nanotube and nanowire based electromechanical systems. App. Phys. Lett. 86(22), 223105 (2005).

T. P. Burg, M. Godin, S. M. Knudsen, W. Shen, G. Carlson, J. S. Foster, K. Babcock and S. R. Manalis. Weighing of biomeolecules, single cells and single nanoparitcles in fluid. Nature. 446, 1066-1069 (2007).

K. L. Ekinci, Y T Yang, M L Roukes. Ultimate limits to inertial mass sensing based upon nanoelectromechanical systems. J. Appl. Phys. 95, 2682-2689 (2004).

M. S. Weinberg, C. E. Dube, A. Petrovich, A. M. Zapata. Fluid damping in resonant flexural plate wave device. J. Microelectromech. Syst. 12, 567-576 (2003).

V. Kumar, J. W. Boley, Y. Yang, H. Ekowaluyo, J. K. Miller, 
G. T-C. Chiu, J. F. Rhoads. Bifurcation-based mass sensing using piezoelectrically-actuated microcantilevers. Appl. Phys. Lett. 98(15), 153510 (2011).

V. Kumar, Y. Yang, J. W. Boley, G. T-C Chiu, J. F. Rhoads. Modeling analysis and experimental validation of a bifurcation-based microsensor. J. Microelectromechan. Syst. 21, 549-558 (2012)

R. H. Harne and K. W. Wang. A bifurcation based coupled linear-bistable system for microscale mass sensing. J. Sound. Vib. 333(8), 2241-2252 (2014).

W J Venstra, M J Capener, S R Elliott. Nanomechanical gas sensing with nonlinear resonant cantilevers. Nanotechnol. 25(42), 425501 (2014).

J. F. Rhoads, S. W. Shaw, K. L. Turner. Nonlinear dynamics and its applications in micro- and nanoresonators. J. Dyn. Syst. Meas. Control. 132, 1509-1538 (2010).

A. Bouchaala, N. Jaber, O. Yassine, O. Shekhah, V. Chernikova, M. Eddaoudi and M. I. Younis. Nonlinear-based MEMS sensors and active switches for gas detection. Sensors. 16(6), 758 (2016).

M. I. Younis, F. Alsaleem. Exploration of new concepts for mass detection in electrostatically-actuated structures based on nonlinear phenomena. J. Comput. Nonlinear Dynam. 4, 710-733 (2009).

V-N. Nguyen, S. Baguet, C-H. Lamarque, and R. Dufour. Bifurcation-based micro/nanoelectromechanical mass detection. Nonlinear Dynam. 79(1), 647-662 (2015).

R. L. Harne, and K. W. Wang. Robust sensing methodology for detecting change with bistable circuitry dynamics tailoring. Appl. Phys. Lett. 102, 203506 (2013).

W. Zhang; K. L. Turner. Application of parametric resonance amplification in a single-crystal silicon microoscillator based mass sensor. Sens. Actuators A Phys. 122, 23-30 (2005).

N. Kacem, S. Baguet, R. Dufour, S. Hentz. Overcoming limitations of nanomechanical resonators with simultaneous resonances. Appl. Phys. Lett. 107, 583 (2015).

G. Prakash, A. Raman, J. Rhoads, and R. G. Reifenberger. Parametric noise squeezing and parametric resonance of microcantilevers in air and liquid environments. Rev. Sci. Instrum. 83, 065109 (2012).

L. Li, E. Holthoff, L. Shaw, C. Burgner, and K. Turner. Noise squeezing controlled parametric bifurcation tracking of MIP-coated microbeam MEMS sensor for TNT explosive gas sensing. J. Microelectromech. Syst. 23(5), 1228-1235 (2014).

G. Chen, F. Ma. Kinetostatic Modeling of Fully Compliant Bistable Mechanisms Using Timoshenko Beam Constraint Model. J. Mech. Design. 137(2), 022301 (2015).

Q. Han, K. Jin, G. Chen, X. Shao. A novel fully compliant tensural-compresural bistable mechanism. Sensor Actuat.APhys. 268, 72-82(2017).

M. Gurjar and N. Jalili. Toward ultrasmall mass detection using adaptive self-sensing piezoelectrically driven microcantilevers. IEEE/ASME Trans. Mechatron. 12(6), 680-688 (2007).

K. L. Ekinci, Y. T. Yang, and M. L. Roukes. Ultimate limits to inertial mass sensing based upon nanoelectromechanical systems. J. App. Phys. 95(5), 2682-2689 (2004).

W. P. Robins Phase Noise in Signal Sources. Peter Peregrinus LTD, 1982.

N. Kacem, S. Hentz, D. Pinto, B. Reig, and V. Nguyen. Nonlinear dynamics of nanomechanical beam resonators: improving the performance of NEMS-based sensors. Nanotechnol. 20, 275501 (2009).

C. Stambaugh, H. Chan. Noise-activated switching in a driven nonlinear micromechanical oscillator, Phys. Rev. B. 73,172302 (2006).

H. B. Chan, C. Stambaugh. Activation barrier scaling and crossover for noise-induced switching in micromechanical parametric oscillators. Phys. Rev. Lett. 99, 060601 (2007).

M. I. Dykman, M. Khasin, J. Portman, S. W. Shaw. Spectrum of an oscillator with jumping frequency and the interference of partial susceptibilities. Phys. Rev. Lett. 105(23), 060601 (2010).

$\mathrm{M}$ Requa, $\mathrm{K}$ Turner. Precise frequency estimation in a microelectromechanical parametric resonator. Appl. Phys. Lett. 90(17), 173508 (2007)

Z. Yie, M. Zielke, C. Burgner, K. Turner. Comparison of parametric and linear mass detection in the presence of detection noise. J. Micromech. Microeng. 21(2), 025027 (2011). 